This is the post-print version of the following article: Ana Beloqui, and Aitziber L. Cortajarena, Protein-based functional hybrid bionanomaterials by bottom-up approaches, Current Opinion in Structural Biology, 2020, 36/297, 63 (74-81)

DOI: $10.1016 / j . s b i .2020 .04 .005$

This article may be used for non-commercial purposes in accordance with Elsevier Terms and Conditions for Self-Archiving.

(C) $\langle 2020\rangle$. This manuscript version is made available under the CC-BY-NC-ND 4.0 license http://creativecommons.org/licenses/by-nc-nd/4.0/ 
Protein-based functional hybrid bionanomaterials by bottom-up approaches

\author{
Ana Beloqui ${ }^{a, b,}{ }^{*}$, and Aitziber L. Cortajarena ${ }^{b, c,{ }^{*}}$ \\ a POLYMAT and Department of Applied Chemistry, University of the Basque Country \\ UPV/EHU, Avda. Manuel de Lardizabal 3, E-20018 Donostia - San Sebastian (Spain) \\ b IKERBASQUE, Basque Foundation for Science, Maria Diaz de Haro 3, E-48013 Bilbao \\ (Spain) \\ ${ }^{\mathrm{C}} \mathrm{CIC}$ biomaGUNE, Paseo Miramón, 182, E-20014 Donostia - San Sebastian (Spain) \\ *Corresponding authors: alcortajarena@cicbiomagune.es; a.beloqui@nanogune.eu
}

\begin{abstract}
This review aims to summarize the last advances on the field of protein engineering towards functional bionanomaterials. Albeit being this an emerging research field, multidisciplinary perspectives in the design of synthetic protein-based hybrid bionanomaterials have resulted in significant progresses. The review covers the definition of bionanomaterials as such and the description of the main methodological approaches currently employed for their assembly. In this context, special emphasis is placed on the fundamental role of protein design. Then, a general overview of the most recent advances related to the fabrication and application of protein-based bionanomaterials in several applications is provided, with special focus on catalysis. Finally, key aspects to be considered by the research community to establish the path for significant future developments in this promising field are discussed.
\end{abstract}

\title{
Introduction
}

Nature, as the best-known bioengineer, applies smart design strategies for the manufacturing of robust and functional bionanomaterials. Hence, several complex systems have evolved by the combination of biomacromolecules, mostly proteins, with small molecules or inorganic materials, giving rise to the fabrication of advanced and highly efficient hybrids, such as natural photosystems [1], metalloenzymes [2], antenna systems [3], or bionanocomposites [4][5]. In this context, mimicking Nature's complex architectures in the lab arises as a smart strategy for the design of bionanomaterials, by means of interdisciplinary research that compiles latest advances in protein engineering and materials science. In a rational combination of these two disciplines, state-of-theart synthetic approaches will be applied to biomolecules and thereby new opportunities to the increase the functional and/or structural diversity of the bionanomaterials will be opened.

In the last years, the concept of "bionanomaterials" has been applied to an extended number of nanosystems in which at least one of the components is derived from a biological source, such as nucleic acids, carbohydrates, lipids, peptides, and proteins. Herein, we are focused in hybrid materials that are composed by biomolecules, namely 
proteins, and a second component, usually but not restricted to a synthetic material, which combination leads to a single molecular unit which necessarily maintains its dimensions in the nanoscale. Individual components, i.e. proteins and functional elements, must contribute either as a structural platform (scaffold material) or/and as functional component (e.g. catalytic, optical or conductive properties) to the hybrid unit. Proteins, as the main building blocks for bionanomaterials in Nature [6], are the core component of the hybrids that are herein discussed. Rational design and careful engineering of proteins using a multidisciplinary toolbox, based in computational modelling, molecular biology, materials science, and chemistry (Figure 1), has been demonstrated successful towards the development of new functional systems with potential applications in several disciplines including imaging [7], sensing [8], conductive materials [9][10], logic circuits [11], and catalysis [12]. In this review, we will highlight and discuss the recent advances in the field of protein-based hybrid functional bionanomaterials. The two main strategies used for the design of the hybrids, i.e. directconjugation of the components vs. in situ assembly of the hybrid, will be tackled through recent examples and applications of the bionanomaterials, with special focus on applications in catalysis.

\section{Design of Protein Bionanomaterials}

Proteins, besides their significance in Nature, can be exploited in the lab as three dimensional nanoscaffolds with high structural and functional diversity. Thanks to the development in the last decades of a multidisciplinary toolbox that includes protein engineering approaches and computational modeling protein design, very relevant milestones including the design of new protein folds, supramolecular protein assemblies, and activities have been recently achieved [13][14][15][16]. Therefore, it is possible to reach a very strict control over the sequence of the protein to shape the three-dimensional structure and the composition of the amino acids that are exposed to the environment. Indeed, these residues are the main target in the rational design of bionanomaterials. The generation of rational-based site-directed modifications laid out on the surface of the biomolecule allows the precise accommodation of the functional elements, which eventually leads to the design of bionanomaterials with well-arranged architectures [14][17]. The combination of both, proteins and synthetic materials, into a single entity can be performed following several orthogonal bioconjugation methodologies, including non-covalent strong and robust interactions, chemically driven covalent bonds, or physical entrapment [18]. Importantly, the approach selected for the synthesis of the hybrids should guarantee the preservation of the nanoscale and the integrity of individual components within the synthesized bionanomaterial. 


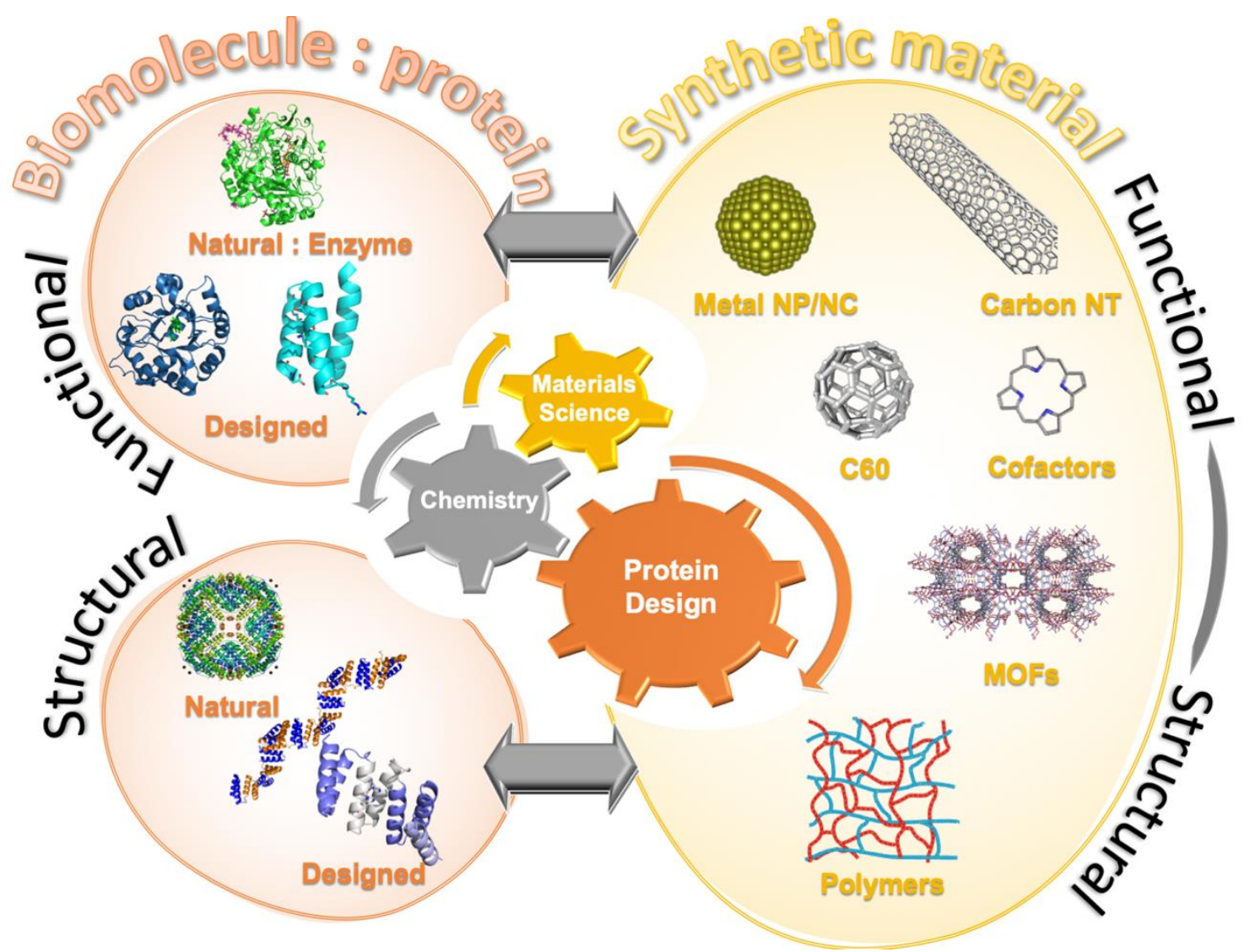

Figure 1. Schematic representation of the main components that may comprise a hybrid functional bionanomaterial, highlighting their role, either structural or functional. The application of a multidisciplinary toolbox that includes recent advances in protein design, materials science, and applied chemistry to the proteins or the synthetic materials, which can operate with a structural or functional role, gives rise to a vast array of possible combinations, and therefore to a large number of potential hybrid bionanomaterials with various structures and functions. The protein component that plays a structural or functional role can currently be selected from an array of natural proteins or from the growing palette of designed proteins. When considering synthetic elements to provide structure or unique functionality, the underlying diversity is equally broad, including nanomaterials, carbon nanomaterials, polymeric materials, metalorganic frameworks, and organic molecules, among others.

The fabrication of hybrid bionanomaterials is mainly performed following two different methodologies, which vary on the assembly procedure that is utilized for the synthesis of the functional element. In a first approach, well-characterized functional elements are combined with proteins using bioconjugation techniques. Here, exposed reactive amino acids or hydrophobic patches on the surface of the protein are usually targeted and the proteins eventually remain anchored to the surface of the functional element [18]. Thus, the main role of the synthetic material here is to act as a scaffolding support, usually to concentrate and orientate functional proteins, such as enzymes or signal peptides. This methodology is widely used in the fabrication of protein-metal core-shell nanoparticles for biomedical applications [19], or in the generation of enzyme-powered nanomotors for active and targeted drug delivery [20]. However, in other examples, the rational design of proteins as scaffolding units can additionally support and stabilize 
functional elements such as carbon nanomaterials, including carbon nanotubes[21] and fullerenes [22][23], and photo-active elements [24][21] by the introduction of selective coordinating sites, giving rise to hybrid bionanomaterials with interesting properties for bioelectronics.

The second strategy for the synthesis of bionanomaterials includes the assembly of the functional element in presence of the protein, meaning that as the material grows, the protein hybrid is synthesized. In the last years, several technologies for the in-situ synthesis of protein hybrid bionanomaterials have been developed. Compared to the direct-conjugation approach described above, the in situ synthesis allows facile one-pot synthetic protocols that determine the location of the nanomaterial in specific sites within the protein, while preserving the nanoscale size of the hybrid. As a typical example of bottom-up synthesis approach, proteins have been used as platforms for the nucleation of metal ions and subsequent growth of metal nanoparticles on the surface of the biomolecule. Both structural, i.e. ferritin [25], or functional, i.e. enzymes [26], native proteins have been employed as scaffolds for the controlled synthesis of catalytic metal nanoparticles. However, from the protein design perspective, there are few reported examples in which the protein sequence is carefully tailored to obtain a thorough control of the growth of the nanomaterials. In a pioneering work Ueno et al. modified the ferritin cage for the nucleation of sub-nanoclusters [27]. These small-sized clusters could be only achieved by the creation of a controlled chemical environment through the manipulation and accommodation of the cavity of the protein. Recently, a protein-engineering based approach has been established for the use of protein scaffolds to control in situ synthesis of metal nanomaterials by the introduction of specific metal coordination sites in well-defined cavities on the protein surface [28][29]. In addition, this well-controlled bottom-up approach presents the advantage of the modularity of the protein scaffold of choice, a repeat protein domain, that allows the modular design of the scaffold protein and thus the precise control of the size and properties of the final bionanomaterial [28][30].

Another interesting and cutting-edge example of protein-hybrid nanomaterials synthesized through in situ assembly is based on the encapsulation of proteins in metal organic frameworks (MOFs) [31]. Compared to the post-conjugation strategy, the in situ approach, besides showing more conclusive results in the stabilization of proteins and delivery applications, keeps the small size of the hybrids. In this bottom-up synthesis strategy, the entrapment of the proteins in MOFs shell is triggered by the hydrogen and ionic interactions between the residues of the surface of the protein and the coordination ligands and metal cations that build up the functional element starting from the surface of the protein. Therefore, the chemical composition of the surface of the biomolecule is again key for the assembly of the protein-MOF hybrids. This feature is clearly evidenced in the works of Prof. Doonan [32] and Prof. Ouyang [33], in which the effect of different chemical environments on the surface of the protein in the embedment of the proteins into a MOF exoskeleton were studied.

Alternatively, polymers are excellent platforms to host biomolecules. Polymer-based bionanomaterials have desirable properties such as solubility, functionality, enhanced stability of the protein against denaturation, thermo-responsiveness, or conductivity, 
being all features provided by the synthetic material [34]. Indeed, it has been demonstrated that the combined polymer-protein hybrids show a synergistic effect that benefits both counterparts, overcoming their inherent limitations [35][36]. Polymerprotein conjugates (PPCs) can be classified according to the conjugation technique used in the synthesis (e.g. covalent bond, ionic interaction, supramolecular interaction, or physical entrapment of the protein) or to the architecture of the hybrid (e.g. dendrimers, polymer nanoparticles - from solid nanoparticles to polymersomes -, or grafted copolymers) [37]. Among all the diverse architectures and chemistries that have been brought to the table in the last years in this field, we want to emphasize the use of single enzyme nanogels (SENs) as polymer-based bionanomaterials with enormous potential in sensing, biocatalysis, and delivery applications [38][39]. SENs are synthesized in situ by the action of acrylic polymers on the surface of the protein. The synthesis of such small nanogels relies on the nature of the surface exposed chemical groups on the protein [40]. This method leads to the synthesis of a very thin polymeric layer that wraps single proteins, which remain in the core of the so-called single protein nanogels, keeping, in this way, the nanoscale dimensions of the bionanomaterial [41].

Herein, it is essential to remark, regardless the strategy of choice, that a particular emphasis is needed on preserving protein structure and function throughout the synthesis processes. All the utilized protocols and methods will be thereby conditioned to the stability of the protein in order to guarantee the success of the protein-based bionanomaterial fabrication and the usefulness of these for a desired application.

\section{Protein hybrid bionanomaterials for catalysis}

The use of enzymes and/or catalytic nanomaterials, such as metal nanoparticles and organometallic complexes, as components of the biohybrids enables their use as catalysts. In this context, the use of tailored bionanohybrids opens the scope to the development of non-natural catalysis. These can be achieved by two approaches: (1) the generation of artificial protein-based biocatalysts combining protein design and the coordination with catalytic elements; and (2) the application of protein-based catalysts (enzymes) and their integration in bionanomaterials which can be composed of different structural elements including proteins. Furthermore, the combination of bio- and chemo-catalysts in a single unit opens new opportunities towards the fabrication of multifunctional materials. In addition, the assembly of protein-hybrid bionanomaterials into macroscopic materials, such as films and biocoatings, gives rise to several advantages compared to stablished technologies in homogeneous and conventional heterogeneous catalysis. Indeed, assembled bionanomaterials, as heterogeneous materials, enhance the processability, robustness, and stability of the catalysts [42] [43].

A very integrative example of bionanohybrids synthesized using the first approach consists in the design and development of the artificial metalloenzymes (ArM). A straightforward methodology uses small proteins with non-covalently bound cofactors, mostly hemin proteins such as cytochrome $\mathrm{C}$ (CytC) or myoglobin (Mb). Small proteins are easier to manipulate, and the removal of the hemin cofactor leaves a well-defined cavity to introduce the engineered cofactor. As example, Hayashi et al. [44] successfully designed and synthesized an ArM with hydrogenase activity by the introduction of a 
[2Fe-2S] type H-cluster to the apo-CytC protein. The same group has reconstituted apo$\mathrm{Mb}$ with manganese porphycene 7 for the hydroxylation of alkanes using $\mathrm{H}_{2} \mathrm{O}_{2}$ [44].

In a more advanced approach, protein scaffolds are sought in the protein data bank and selected according to their suitability for the catalysis. In this case, the exposed residues of selected scaffolds are carefully tailored to accommodate organic catalysts, mostly metalorganic cofactors. Such modifications are usually performed on existing pockets on the surface of the proteins to anchor the functional elements specifically using orthogonal chemistries. Further, strategic sites of the surface, i.e. substrate channels, of the protein can be modified to modulate the selectivity, and thus functionality, of the ArM. Engineered scaffolds have been used for the synthesis of artificial oxidases, hydrolases or Diels-Alderases, to cite some examples [45][46]. Interestingly, second generation ArMs are achieved by the introduction of random mutations to the protein sequence through directed evolution cycles. Hence, recent works report the possibility to modulate the chemo-, stereo-, and regio-selectivity of the organic catalysts by random modification of the protein scaffolds in which are anchored [47].

In a different approach from the methodological point of view, synthetic materials can be used as scaffolds of hybrid nanosystems in which proteins provide the functionality. Usually, these non-biological systems are used either to protect the proteins from denaturation under non-physiological conditions or to arrange and confine them into favorable environments for catalysis. As explained above, the use of polymers as synthetic component of the hybrids is of high interest not only due to the new features that polymers provide, but also due to the high chemical diversity that the polymers bring to the hybrid systems. In this regard, the single enzyme nanogel approach allows the modification of the surface of the proteins and the introduction multiple coordination ligands on the protein in a delocalized manner but skipping the molecular biology steps. As result of the high modification density, low concentration of metal cations is needed for the efficient assembly of enzyme nanogels into robust and highly active nanoparticles, so-called Metal-Organic Enzyme Aggregates (MOEAs) [48]. These hybrids have been demonstrated successful not only as new bifunctional biocatalysts that integrate the co-catalysis of the biomolecule and the metal cation in a synergy action, but also as assembled platforms to physically compartmentalize biocatalysts in enzymatic cascade reactions and to coat gold microelectrodes for the electrochemical detection of glucose [43].

In contrast to classical approaches to assemble nanomaterials into macroscopic materials, usually based in chemically driven crosslink, there are some proteins that can naturally undergo self-assembly through only protein-protein interactions. These proteins include natural proteins mostly silk fibroin [49], and designed proteins, mostly based on repeat proteins [50]. The self-assembly of engineered protein scaffolds has been applied to the fabrication of protein-based macroscopic materials that entrap and stabilize enzymes resulting in the generation of novel fully protein-based heterogeneous biocatalysts [51][52]. In addition, the ability of the certain engineered proteins to selfassemble has been also demonstrated when using protein-hybrids that carry additional functional elements such as photoactive elements [53], redox active clusters [28], and conductive materials [21], which set the basis to explore this technology to fabricate other biocatalytic materials in which the catalytic entity is not a protein. 


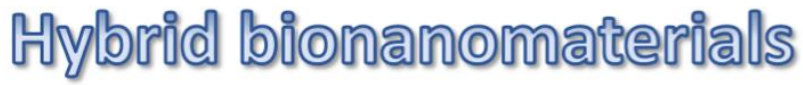

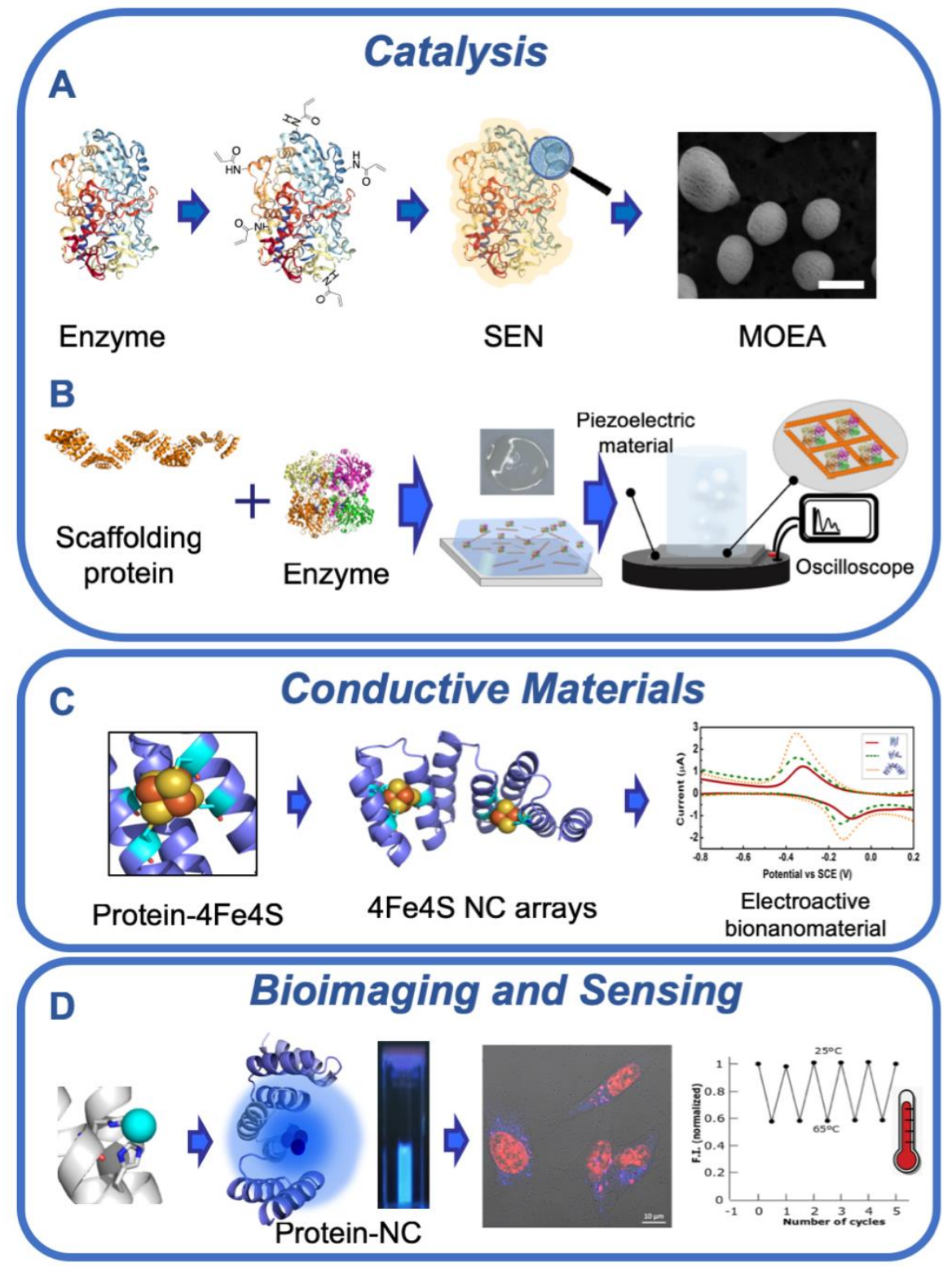

Figure 2. Representative examples of different protein-hybrid bionanomaterials assemblies for applications in catalysis, bioelectronics, bioimaging, and biosensing. A. Example of protein as functional element of a protein-nanogel engineered bionanomaterial in which a polymer acts as a structural element [48]. B. Example of fully protein-based functional nanostructured biocatalytic material in which an engineered scaffold protein plays a structural role and an enzyme is the functional element [51][52]. C. Example of designed protein for the stabilization of ordered arrays of redox-active clusters, for the modular design of long range electron transfer conduits [28]. D. Example of protein as designed structural scaffold that templates functional fluorescent metal nanomaterials that can be used for in cell bioimaging [29] and sensing [54], for example as temperature sensors. 
As a last combination strategy for the fabrication of catalytic bionanomaterials, there are few examples of complex hybrids in which functional biomolecules and functional elements are merged in a single unit, i.e. enzymes with (in)organic catalysts [26]. Being this a good strategy for, as example, the development of chemoenzymatic reactions, in the practice, these multifunctional hybrids are usually not operative or achieved low reaction efficiencies. There are some issues that preclude the use of both catalysts at the same time, such as the cross-poisoning or the excluding working conditions of each of the elements. Gratifyingly, successful stories have been reported by the combination of (bio)catalysts with a support material. However, the latter materials, e.g. mesoporous silica materials or UiO-66 based MOFs, are usually macroscopic, thereby being no longer considered bionanomaterials [55]. Only recently, Li et al. developed a method for the fabrication of enzyme - metal nanoparticle bionanohybrids using enzyme-polymer conjugates [56]. They claimed the controlled synthesis of Pd nanoparticles on a polymer matrix that is bound to the CALB enzyme. With this strategy, they demonstrated the chemoenzymatic production of enantiomerically pure alcohols and amines via a Dynamic kinetic resolution (DKR) process.

In our view, future directions in the field of protein design point towards the achievement of fully synthetic catalytic systems. In this direction, engineered artificial proteins based on repeat proteins were shown to catalyze a $(3+2)$ cycloaddition, a reaction not described to be catalyzed by any natural enzyme [57]. In addition, similarly to the synthesis of ArMs, protein-metal cluster hybrids with potential catalytic activity have been designed using the same repeat scaffolding protein [29]. These atomically precise metal nanoclusters present interesting catalytic properties mostly due to their high surface area and high surface reactivity. Therefore, the aforementioned proteindesign strategies reveal the possibility of developing in the near future fully designed chemoenzymatic catalysts. For example, by modular combination in the same bionanomaterial of a fully designed enzyme and an artificial ArM with inorganic components all based on similar scaffolding proteins and on the precise bottom-up assembly of individual functional components into a la carte complex systems. Those strategies present high promise for the next generation of tunable bionanocatalysts and the achievement from simple strategies of a complexity and functional diversity currently not imaginable in single bionanomaterials. Overall, it seems that there is an interesting open field in the research of new approaches for the design of bionanomaterials that overcome the limitations herein exposed and, in turn, enable the accommodation of different functional elements at the nanoscale.

\section{Protein hybrid bionanomaterials for other applications}

The use of functional proteins, i.e. enzymes, and catalytic elements leads the use of bionanomaterials as excellent opportunities in the field of catalysis. However, this is not the only field of action in which the hybrids can contribute with significant advances. The achievement of protein stabilized small (around $2 \mathrm{~nm}$ ) metal-nanoclusters (NCs) and metal-nanoparticles (NPs) allows their use as sensors owing to the responsiveness of those to temperature or the presence of reactive oxygen (ROS) species [8][54], as 
surface enhanced raman scattering (SERS) substrates [58], and as robust tools for bioimaging [29]. In addition, engineered proteins have been used to achieve a precise control over the organization of photoconductive elements, i.e. porphyrin and single wall carbon nanotubes (SWCNT) [21], or conductive metal centers [9][28], that give rise to the fabrication of new and sophisticated materials with enhanced properties in the field of bioelectronics.

\section{Conclusions and future perspectives}

As discussed in this review, significant progresses have been recently made in the generation of protein-hybrid bionanomaterials. These bionanomaterials as defined above should have nanoscale dimensions, a protein element that can act as a functional or structural component, and a second synthetic element that also can provide either structure or function to the hybrid. Thus, the generation protein-hybrid bionanomaterials aims to expand the potential of the already versatile proteins by mixing proteins with other functional or structural elements towards hybrid systems that encode even larger structural and functional complexity than proteins by themselves. The examples shown here illustrate the versatility and emerging potential of these bionanomaterials in terms of achieving a huge range of structures and functions. Even though the review mostly focused on examples in catalysis, it is worth mentioning that the approaches presented have general interest and a broad range of potential applications.

New approaches are being applied for the development and design of new bionanomaterials based on proteins. The significance of using proteins in the fabrication of bionanomaterials goes beyond their intrinsic functionality, since proteins can also be used as highly tunable platforms, as scaffolds, for the accommodation and tethering of synthetic materials that bring new functionalities to the hybrid system. In addition to this functional and structural versatility of proteins as design building blocks, it should be noted that compared to other platforms, protein-based materials are green, sustainable, biodegradable, and biocompatible. Therefore, it becomes clear the impact that these approaches will make on the development of new, sustainable, eco-efficient, and competitive functional materials contributing to the required transition to a biobased economy.

The in situ fabrication of functional bionanomaterials is a current challenge that has already shown great future potential. The development of new tools and the integration of different disciplines in the study of bionanomaterials has led to the achievement of new materials that can be "easily" tailored to achieve fully encodable properties. In this regard, the research on fundamental protein design, including focus on structure, functionality, and directed and precise localization at molecular level of functionalization sites by applying structure-based design, will be essential to achieve a high control of the supramolecular structure of the bionanomaterials. The high complexity of the interactions between materials of different nature should be also understood, especially in organic-inorganic complex hybrids. When precise control is not required, eg. when the surface of the biomolecule is fully modified, the supramolecular assembly and growth on the surface of the biomolecules is an attractive 
and simpler approach for the synthesis of functional hybrids. For the short-term success and implementation of these technologies, integrative approaches and continuous feedback between research disciplines are required. This combined approach will bring us the possibility to design "a la carte" bionanomaterials, with rational designs and fully encodable properties for specific requirements.

\section{Acknowledgements}

A.L.C. acknowledges the European Research Council ERC-CoG-648071-ProNANO, ERCPoC-2018-841063-NIMM, the Spanish Ministry of Economy and Competitiveness (BIO2016-77367-R), and the Basque Government (Elkartek KK-2017/00008). A.L.C. also acknowledges the Maria de Maeztu Units of Excellence Program from the Spanish State Research Agency - Grant No. MDM-2017-0720 (CIC biomaGUNE). A.B acknowledges the Spanish Ministry of Economy and Competitiveness (MAT2017-88808-R) and the Agencia Estatal de Investigación and Fondo Social Europeo for Ramón y Cajal Program (RYC2018025923-I).

\section{References and recommended reading}

Papers of particular interest, published within the annual period of review, have been highlighted as:

- of special interest

$\bullet$ of outstading interest

1. Suga $M$, Qin $X$, Kuang $T$, Shen J-R: Structure and energy transfer pathways of the plant photosystem I-LHCl supercomplex. Curr Opin Struct Biol 2016, 39:46-53.

2. Valdez CE, Smith $Q A$, Nechay $M R$, Alexandrova AN: Mysteries of metals in metalloenzymes. Acc Chem Res 2014, 47:3110-3117.

3. Mirkovic T, Ostroumov EE, Anna JM, van Grondelle R, Govindjee null, Scholes GD: Light Absorption and Energy Transfer in the Antenna Complexes of Photosynthetic Organisms. Chem Rev 2017, 117:249-293.

4. Hu X, Cebe P, Weiss AS, Omenetto F, Kaplan DL: Protein-based composite materials. Mater Today 2012, 15:208-215.

5. Frezzo JA, Montclare JK: Natural Composite Systems for Bioinspired Materials. Adv Exp Med Biol 2016, 940:143-166.

6. Abascal NC, Regan L: The past, present and future of protein-based materials. Open Biol 2018, 8.

7. Berwick MR, Slope LN, Smith CF, King SM, Newton SL, Gillis RB, Adams GG, Rowe AJ, Harding SE, Britton MM, et al.: Location dependent coordination chemistry and 
MRI relaxivity, in de novo designed lanthanide coiled coils. Chem Sci 2016, 7:22072216.

8. Couleaud P, Adan-Bermudez S, Aires A, Mejías SH, Sot B, Somoza A, Cortajarena AL: Designed Modular Proteins as Scaffolds To Stabilize Fluorescent Nanoclusters. Biomacromolecules 2015, 16:3836-3844.

9. Altamura L, Horvath C, Rengaraj S, Rongier A, Elouarzaki K, Gondran C, Maçon ALB, Vendrely $\mathrm{C}$, Bouchiat $\mathrm{V}$, Fontecave $\mathrm{M}$, et al.: A synthetic redox biofilm made from metalloprotein-prion domain chimera nanowires. Nat Chem 2017, 9:157-163.

- This work described the development of a conductive nanowire consisting solely of proteins that combines a self-assemble unit based on a prion domain and a protein domain with redox metal centres that act as electron carriers. This approach opens opportunities for the engineering of protein-only electron mediators and applications in the field of protein-based bioelectronics.

10. Gogurla N, Sinha AK, Naskar D, Kundu SC, Ray SK: Metal nanoparticles triggered persistent negative photoconductivity in silk protein hydrogels. Nanoscale 2016, 8:7695-7703.

11. Medalsy I, Klein M, Heyman A, Shoseyov O, Remacle F, Levine RD, Porath D: Logic implementations using a single nanoparticle-protein hybrid. Nat Nanotechnol 2010, 5:451-457.

12. Keller D, Beloqui A, Martínez-Martínez M, Ferrer M, Delaittre G: Nitrilotriacetic Amine-Functionalized Polymeric Core-Shell Nanoparticles as Enzyme Immobilization Supports. Biomacromolecules 2017, 18:2777-2788.

13. Sasaki E, Böhringer D, van de Waterbeemd $M$, Leibundgut $M$, Zschoche $R$, Heck AJR, Ban N, Hilvert D: Structure and assembly of scalable porous protein cages. Nat Commun 2017, 8:14663.

14. Ross JF, Bridges A, Fletcher JM, Shoemark D, Alibhai D, Bray HEV, Beesley JL, Dawson WM, Hodgson LR, Mantell J, et al.: Decorating Self-Assembled Peptide Cages with Proteins. ACS Nano 2017, 11:7901-7914.

15. Langan RA, Boyken SE, Ng AH, Samson JA, Dods G, Westbrook AM, Nguyen TH, Lajoie MJ, Chen Z, Berger S, et al.: De novo design of bioactive protein switches. Nature 2019, 572:205-210.

- In this work Baker and co-workers achieve the de novo design of switchable proteinbased cages. The ability to design switchable protein functions that are controlled by induced conformational change is a milestone for de novo protein design, and opens up new avenues for engineering of switchable protein-based bionanomaterials.

16. Yeates TO: Geometric Principles for Designing Highly Symmetric Self-Assembling Protein Nanomaterials. Annu Rev Biophys 2017, 46:23-42. 
17. Liljeström V, Ora A, Hassinen J, Rekola HT, Nonappa null, Heilala M, Hynninen V, Joensuu JJ, Ras RHA, Törmä $P$, et al.: Cooperative colloidal self-assembly of metalprotein superlattice wires. Nat Commun 2017, 8:671.

18. Biju V: Chemical modifications and bioconjugate reactions of nanomaterials for sensing, imaging, drug delivery and therapy. Chem Soc Rev 2014, 43:744-764.

19. Spicer CD, Jumeaux C, Gupta B, Stevens MM: Peptide and protein nanoparticle conjugates: versatile platforms for biomedical applications. Chem Soc Rev 2018, 47:3574-3620.

20. Hortelão AC, Carrascosa R, Murillo-Cremaes N, Patiño T, Sánchez S: Targeting 3D Bladder Cancer Spheroids with Urease-Powered Nanomotors. ACS Nano 2019, 13:429-439.

21. López-Andarias J, Mejías SH, Sakurai T, Matsuda W, Seki S, Feixas F, Osuna S, Atienza C, Martín N, Cortajarena AL: Toward bioelectronic nanomaterials: photoconductivity in protein-porphyrin hybrids wrapped around SWCNT. Adv Funct Mater 2018, 28:1704031.

22. Kim K-H, Ko D-K, Kim Y-T, Kim NH, Paul J, Zhang S-Q, Murray CB, Acharya R, DeGrado $\mathrm{WF}$, Kim $\mathrm{YH}$, et al.: Protein-directed self-assembly of a fullerene crystal. Nat Commun 2016, 7:1-9.

23. Liutkus M, López-Andarias A, Mejías SH, López-Andarias J, Gil-Carton D, Feixas F, Osuna S, Matsuda W, Sakurai T, Seki S, et al.: Protein-Directed Crystalline 2D Fullerene Assemblies. Nanoscale 2019, doi:10.1039/c9nr07083d.

24. Polizzi NF, Wu Y, Lemmin T, Maxwell AM, Zhang S-Q, Rawson J, Beratan DN, Therien MJ, DeGrado WF: De novo design of a hyperstable non-natural protein-ligand complex with sub-Å accuracy. Nat Chem 2017, 9:1157-1164.

25. Wang Z, Gao H, Zhang Y, Liu G, Niu G, Chen X: Functional ferritin nanoparticles for biomedical applications. Front Chem Sci Eng 2017, 11:633-646.

26. Palomo JM: Nanobiohybrids: a new concept for metal nanoparticles synthesis. Chem Commun 2019, 55:9583-9589.

27. Maity $B$, Abe $S$, Ueno $T$ : Observation of gold sub-nanocluster nucleation within a crystalline protein cage. Nat Commun 2017, 8:1-9.

28. Mejias SH, Bahrami-Dizicheh Z, Liutkus M, Sommer DJ, Astashkin A, Kodis G, Ghirlanda G, Cortajarena AL: Repeat proteins as versatile scaffolds for arrays of redox-active FeS clusters. Chem Commun 2019, 55:3319-3322.

29. Aires A, Llarena I, Moller M, Castro-Smirnov J, Cabanillas-Gonzalez J, Cortajarena AL: A Simple Approach to Design Proteins for the Sustainable Synthesis of Metal Nanoclusters. Angew Chem 2019, 58:6214-6219. 
- This work describes a simple approach to design proteins for the sustainable synthesis of highly fluorescent metal NCs by the incorporation of specific metal binding sites. The protein-NCs bionanomaterials are able to enter into living cells, making them useful for live cell imaging and labelling.

30. Geng X, Grove TZ: Repeat protein mediated synthesis of gold nanoparticles: effect of protein shape on the morphological and optical properties. RSC Adv 2015, 5:2062-2069.

31. Liang K, Richardson JJ, Doonan CJ, Mulet X, Ju Y, Cui J, Caruso F, Falcaro P: An Enzyme-Coated Metal-Organic Framework Shell for Synthetically Adaptive Cell Survival. Angew Chem Int Ed 2017, 56:8510-8515.

32. Maddigan NK, Tarzia A, Huang DM, Sumby CJ, Bell SG, Falcaro P, Doonan CJ: Protein surface functionalisation as a general strategy for facilitating biomimetic mineralisation of ZIF-8. Chem Sci 2018, 9:4217-4223.

33. Chen G, Huang S, Kou X, Wei S, Huang S, Jiang S, Shen J, Zhu F, Ouyang G: A Convenient and Versatile Amino-Acid-Boosted Biomimetic Strategy for the Nondestructive Encapsulation of Biomacromolecules within Metal-Organic Frameworks. Angew Chem Int Ed 2019, 58:1463-1467.

34. Ko JH, Maynard HD: A guide to maximizing the therapeutic potential of proteinpolymer conjugates by rational design. Chem Soc Rev 2018, 47:8998-9014.

35. Panganiban B, Qiao B, Jiang T, DelRe C, Obadia MM, Nguyen TD, Smith AAA, Hall A, Sit I, Crosby MG, et al.: Random heteropolymers preserve protein function in foreign environments. Science 2018, 359:1239-1243.

36. Wright TA, Page RC, Konkolewicz D: Polymer conjugation of proteins as a synthetic post-translational modification to impact their stability and activity. Polym Chem 2019, 10:434-454.

- This recent review covers all the approaches and methodologies that are currently used for the synthesis of polymer-protein conjugates, with special focus on stabilization of enzymes.

37. Wu Y, Ng DYW, Kuan SL, Weil T: Protein-polymer therapeutics: a macromolecular perspective. Biomater Sci 2015, 3:214-230.

38. Chen G, Abdeen AA, Wang Y, Shahi PK, Robertson S, Xie R, Suzuki M, Pattnaik BR, Saha K, Gong S: A biodegradable nanocapsule delivers a Cas9 ribonucleoprotein complex for in vivo genome editing. Nat Nanotechnol 2019, 14:974-980.

39. Chapman R, Stenzel MH: All Wrapped up: Stabilization of Enzymes within Single Enzyme Nanoparticles. J Am Chem Soc 2019, 141:2754-2769. 
40. Beloqui A, Baur S, Trouillet V, Welle A, Madsen J, Bastmeyer M, Delaittre G: SingleMolecule Encapsulation: A Straightforward Route to Highly Stable and Printable Enzymes. Small 2016, 12:1716-1722.

41. Beloqui A, Kobitski AY, Nienhaus GU, Delaittre G: A simple route to highly active single-enzyme nanogels. Chem Sci 2018, 9:1006-1013.

42. Rodriguez-Abetxuko A, Morant-Miñana MC, López-Gallego F, Yate L, Seifert A, Knez $M$, Beloqui A: Imidazole-Grafted Nanogels for the Fabrication of Organic-Inorganic Protein Hybrids. Adv Funct Mater 2018, 28:1803115.

43. Rodriguez-Abetxuko A, Sánchez-deAlcázar D, Cortajarena AL, Beloqui A: A Versatile Approach for the Assembly of Highly Tunable Biocatalytic Thin Films. Adv Mater Interfaces 2019, 6:1900598.

44. Oohora K, Onoda A, Hayashi T: Hemoproteins Reconstituted with Artificial Metal Complexes as Biohybrid Catalysts. Acc Chem Res 2019, 52:945-954.

45. Davis HJ, Ward TR: Artificial Metalloenzymes: Challenges and Opportunities. ACS Cent Sci 2019, 5:1120-1136.

46. Doble MV, Jarvis AG, Ward ACC, Colburn JD, Götze JP, Bühl M, Kamer PCJ: Artificial Metalloenzymes as Catalysts for Oxidative Lignin Degradation. ACS Sustain Chem Eng 2018, 6:15100-15107.

47. Reetz MT: Directed Evolution of Artificial Metalloenzymes: A Universal Means to Tune the Selectivity of Transition Metal Catalysts? Acc Chem Res 2019, 52:336-344.

48. Rodriguez-Abetxuko A, Morant-Miñana $M C$, Knez $M$, Beloqui A: Carrierless Immobilization Route for Highly Robust Metal-Organic Hybrid Enzymes. ACS Omega 2019, 4:5172-5179.

- In this paper, a synthetic structural element based on coordination polymers triggers the self-assembly of enzymes giving rise to a new sort of heterogeneous catalysts named Metal Organic Enzyme Aggregates, MOEAs.

49. Huang W, Ling S, Li C, Omenetto FG, Kaplan DL: Silkworm silk-based materials and devices generated using bio-nanotechnology. Chem Soc Rev 2018, 47:6486-6504.

50. Carter NA, Grove TZ: Protein Self-Assemblies That Can Generate, Hold, and Discharge Electric Potential in Response to Changes in Relative Humidity. J Am Chem Soc 2018, 140:7144-7151.

51. Sanchez-deAlcazar D, Velasco-Lozano S, Zeballos N, Lopez-Gallego F, Cortajarena AL: Biocatalytic protein-based materials for integration into energy devices. ChemBioChem 2019, doi:10.1002/cbic.201900047.

52. Sanchez-deAlcazar D, Liutkus M, Cortajarena AL: Immobilization of Enzymes in Protein Films. In Immobilization of Enzymes and Cells: Methods and Protocols / Edition 4/Hardcover. . Springer US; [date unknown]. 
53. Sanchez-deAlcazar D, Romera D, Castro-Smirnov J, Sousaraei A, Casado S, Espasa A, Morant-Miñana MC, Hernandez JJ, Rodríguez I, Costa RD, et al.: Engineered proteinbased functional nanopatterned materials for bio-optical devices. Nanoscale Adv 2019, 1:3980-3991.

54. Aires A, Lopez-Martinez E, Cortajarena A: Sensors Based on Metal Nanoclusters Stabilized on Designed Proteins. Biosensors 2018, 8:110.

55. Ma M, Lu L, Li H, Xiong Y, Dong F: Functional Metal Organic Framework/SiO2 Nanocomposites: From Versatile Synthesis to Advanced Applications. Polymers 2019, 11:1823.

56. Li X, Cao Y, Luo K, Sun Y, Xiong J, Wang L, Liu Z, Li J, Ma J, Ge J, et al.: Highly active enzyme-metal nanohybrids synthesized in protein-polymer conjugates. Nat Catal 2019, 2:718-725.

- This is one of the first examples in which functional proteins and functional elements are successfully combined for concurrent one-pot chemoenzymatic reactions. In this study, a polymeric network is used to host the inorganic catalyst and compartmentalize both (bio)catalysts in a single unit.

57. Rivilla I, Odriozola-Gimeno $M$, Aires A, Gimeno A, Jiménez-Barbero J, TorrentSucarrat M, Cortajarena AL, Cossio FP: Discovering Biomolecules with Huisgenase Activity: Designed Repeat Proteins as Biocatalysts for $(3+2)$ Cycloadditions. J Am Chem Soc 2019, doi:10.1021/jacs.9b06823.

58. Geng X, Leng W, Carter NA, Vikesland PJ, Grove TZ: Protein-aided formation of triangular silver nanoprisms with enhanced SERS performance. J Mater Chem $B$ 2016, 4:4182-4190. 